ROM2F-96/35

July, 1996

hep-th/9607229

\title{
Comments on Gepner Models
}

\section{and Type I Vacua in String Theory}

\author{
Carlo Angelantonj ${ }^{\dagger}$, Massimo Bianchi*, Gianfranco Pradisi*

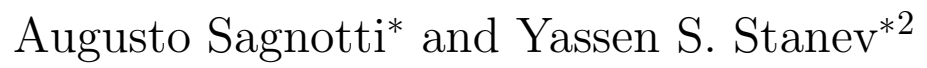 \\ ${ }^{\dagger}$ Dipartimento di Fisica, Università dell' Aquila \\ I.N.F.N. - L.N.G.S. \\ Via Vetoio 67010 Coppito (AQ), ITALY \\ * Dipartimento di Fisica, Università di Roma "Tor Vergata" \\ I.N.F.N. - Sezione di Roma "Tor Vergata", \\ Via della Ricerca Scientifica, 100133 Roma, ITALY
}

\begin{abstract}
We construct open descendants of Gepner models, concentrating mainly on the six-dimensional case, where they give type I vacua with rich patterns of ChanPaton symmetry breaking and various numbers of tensor multiplets, including zero. We also relate the models in $D<10$ without open sectors, recently found by other authors, to the generalized Klein-bottle projections allowed by the crosscap constraint.
\end{abstract}

\footnotetext{
${ }^{1}$ Work supported in part by E.E.C. Grant CHRX-CT93-0340.

${ }^{2}$ I.N.F.N. Fellow, on Leave from Institute for Nuclear Research and Nuclear Energy, Bulgarian Academy of Sciences, BG-1784 Sofia, BULGARIA.
} 


\section{Introduction}

Superstring propagation on manifolds of $S U(n)$ holonomy requires extended world-sheet superconformal symmetry [1]. Gepner has shown how to describe such vacua using tensor products of $N=2$ superconformal minimal models [2]. The underlying Calabi-Yau (CY) manifolds are complete intersections in weighted projective space [3]. In principle, mirror symmetry allows the computation of world-sheet instanton corrections [4] to the low energy effective lagrangian. More recently, second-quantized mirror symmetry [5] has opened the way to computations of non-perturbative string corrections for $N=2$ heterotic - type II dual pairs [6]. The extension of these results to $N=1$ models calls for a better understanding of the relation between heterotic and type I vacua. The conjectured 10D duality between the $S O(32)$ heterotic string and the type I superstring [7] has passed several tests [8], and is expected to persist, in some non-naive form, after compactification [9]. In this respect it is worth noticing that the relation between type I and heterotic dilatons depends on the space-time dimension $D$ according to [10]

$$
\phi_{H}=\frac{6-D}{4} \phi_{I}-\frac{D-2}{16} \log \operatorname{det} G_{I},
$$

where $G_{I}$ is the internal metric in the type I string-frame. For instance, in six dimensions the heterotic dilaton is related to internal gravitational modes of the type I string, rather than to the type I dilaton [11].

In the past, type I models have been studied to a lesser extent than models of oriented closed strings. The initial proposal [12] of identifying open-string theories as parameterspace orbifolds of left-right symmetric theories of oriented closed strings has been brought to a consistent systematization [13, 14, realizing Chan-Paton (CP) symmetry breaking at the microscopic level. The first consistent 6D $N=1$ chiral open-string models [13] differ markedly from perturbative heterotic K3 compactifications [15, 2, 16], since they include different numbers of tensor multiplets that take part in a generalized Green-Schwarz (GS) mechanism [17]. Recently, additional instances of $N=1$ type I 6D models have been constructed as toroidal orbifolds [18, [19], along the lines of [20]. A nice geometrical setting for all these 6D models is provided by the F-theory proposal of [21], where non- 
trivial scalar backgrounds allow for an effective $12 \mathrm{D}$ dynamics. The variety of $6 \mathrm{D}$ models with different numbers of tensor multiplets may then be related to a corresponding variety of compactifications on elliptically-fibered CY threefolds [22].

In this letter we discuss descendants of type IIB models based on rational superconformal field theories. We mainly concentrate on $6 \mathrm{D}$ models, where a precise comparison with orbifold compactifications can be drawn. This allows a complementary view of several known results, while introducing a novelty, models with no tensor multiplets at all. Here we discuss only the simplest Gepner models, related to free conformal field theories. We also relate the models without an open sector of [19] to the "crosscap constraint" of [24, 25]. This consistency condition for conformal field theories on open and unoriented surfaces allows, in general, multiple choices for the Klein-bottle projection of the closed spectrum. For minimal and $S U(2)$ WZW models, this was discussed in [25]. For sixdimensional models, it retrieves the unique anomaly-free $N=1$ spectrum with 9 tensor multiplets and 12 hypermultiplets found in [19. More complicated models, including $N=1$ type I models in four dimensions, can be analyzed in a similar fashion and are full of interesting surprises. For instance, starting from the $Z$ orbifold, one can build

chiral $N=1$ type I vacua in $\mathrm{D}=4$ [10], where the moduli space of untwisted closed-string scalars, $S p(8) / S U(4) \times U(1)$, is strongly suggestive of a twelve-dimensional interpretation [26]. A more complete analysis of rational superconformal models and of some of their deformations will be presented elsewhere [28].

\section{Open Descendants of Gepner Models}

In addition to the Virasoro generators $L_{n}$, the $N=2$ superconformal algebra includes two supercurrents $G_{r}^{ \pm}$and a $U(1)$ current $J_{n}$. It admits an automorphism, known as spectral flow, under which

$$
L_{n} \rightarrow L_{n}+\eta J_{n}+\frac{\eta^{2} c}{6} \delta_{n, 0}, \quad J_{n} \rightarrow J_{n}+\frac{\eta c}{3} \delta_{n, 0}, \quad G_{r}^{ \pm} \rightarrow G_{r \pm \eta}^{ \pm}
$$

where $0 \leq \eta<1$ and $c$ is the central charge, that connects different sectors of the spectrum. In models describing superstring propagation, the coupling to the $N=1$ 
world-sheet gravitino induces a restriction to the Ramond (R) ( $G$-periodic) and NeveuSchwarz (NS) (G-anti-periodic) sectors. The primary fields of the $N=2$ superconformal algebra are labeled by the conformal weight $h$ and the $U(1)$ charge $q$. In the NS sector, the massless vertex operators involve chiral primary fields with $h=q / 2$. Their supersymmetric partners in the $\mathrm{R}$ sector, with $h=c / 24$, arise from the spectral flow with $\eta=1 / 2$. The unique $\mathrm{R}$ descendant of the identity $(h=0)$ is identified with the target-space supercharge.

Gepner has shown how to construct string vacua with spacetime supersymmetry tensoring $N=2$ superconformal minimal models [2], that form a discrete series with central charge $c_{k}=3 k /(k+2)$. For $d=(D-2)$ non compact (transverse) dimensions, the total internal central charge is $c_{I}=\sum_{i} c_{k_{i}}=12-3 d / 2$, where $c_{k_{i}}$ are the central charges of the various factors. The correct periodicity of the world-sheet supercurrent is ensured if characters from the NS and R sectors are combined separately. Target-space supersymmetry follows if the supercharge has a local operator product expansion with all vertex operators. The required truncation ( $G$-projection), induced by the restriction to integral $U(1)$ charges, is consistent with modular invariance if $c_{I}=3 n$, and the "orbifoldized" tensor product of $N=2$ minimal models then describes superstring propagation on a manifold of $S U(n)$ holonomy.

The general procedure for constructing type IIB vacua, described in [16], starts with the identification of the graviton orbit in the NS sector, $N_{0}^{+}$. The other massless $(m)$ and massive $(M)$ NS orbits, $N_{\alpha}^{+}, \alpha=1, \ldots, n_{m}+n_{M}$, are exposed by a modular $S$ transformation on $N_{0}^{+}$. Their projections $N_{\alpha}^{-}$are obtained by a $T$ transformation, while the $\mathrm{R}$ orbits $R_{\alpha}^{+}$are obtained by an $S$ transformation from $N_{\alpha}^{-}$. Finally, a spectral flow with $\eta=1$ determines the projected $\mathrm{R}$ orbits, $R_{\alpha}^{-}$. Introducing the supersymmetric characters

$$
X_{\alpha}=\frac{1}{2}\left(V_{d}\left(N_{\alpha}^{+}+N_{\alpha}^{-}\right)+O_{d}\left(N_{\alpha}^{+}-N_{\alpha}^{-}\right)-C_{d}\left(R_{\alpha}^{+}-R_{\alpha}^{-}\right)-S_{d}\left(R_{\alpha}^{+}+R_{\alpha}^{-}\right)\right),
$$

where $\left\{V_{d}, O_{d}, C_{d}, S_{d}\right\}$ are level-one $S O(d)$ characters, a modular invariant partition func- 
tion with space-time supersymmetry can be written as

$$
\mathcal{T}_{\text {susy }}=\sum_{\alpha=0}^{n_{m}+n_{M}} \ell_{\alpha}\left|X_{\alpha}\right|^{2}
$$

where $\ell_{\alpha}=S_{0 \alpha} / S_{\alpha 0}$ are the multiplicities of each orbit. In order to construct open descendants of L-R symmetric closed models as in [13], one has to resolve the fixedpoint ambiguity in the definition of the characters. The different modular invariant torus amplitudes may be related to the propagation on different complex manifolds, consistently with the ADE classification of simple singularities [3].

\section{$3 \quad \mathrm{D}=8$ and Other Toroidal Compactifications}

Gepner models with $c=3$ describe compactifications on rational tori. For instance, the orbifoldized tensor products $(k=1)^{3}$ and $(k=1) \times(k=4)$ correspond to the $S U(3)$ torus, while $(k=2)^{2}$ corresponds to the $S U(2) \otimes S U(2)$ torus [2]. The resulting open descendants are among the rational toroidal compactifications discussed in [14]. There it was shown how a quantized background for the NS-NS antisymmetric tensor reduces the size of the CP group and how CP symmetry breaking may proceed via Wilson lines. This setting plays a crucial role in establishing a correspondence between F-theory on K3 and heterotic string on $T_{2}$ [23.

In general, the Klein bottle amplitude allows for the introduction of signs $\epsilon_{i}= \pm 1$ in the projection of the closed-string spectrum. This was discussed in detail for $S U(2)$ WZW models in [25]. In a generic $r$-dimensional toroidal compactification at radii $R_{i}$, with $i=1, \ldots, r$, it is possible to choose a Klein bottle amplitude (neglecting irrelevant factors)

$$
\mathcal{K}=\prod_{i=1}^{r}\left(\sum_{m_{i} \text { even }} q^{\left(\frac{m_{i}}{R_{i}}\right)^{2}}+\epsilon_{i} \sum_{m_{i} \text { odd }} q^{\left(\frac{m_{i}}{R_{i}}\right)^{2}}\right),
$$

where the conventional choice is $\epsilon_{i}=1$. When at least one of the $\epsilon_{i}$ equals -1 , there are no massless tadpoles, and thus one can not introduce boundary states and open strings. From a microscopic viewpoint, the consistency of this choice may be justified from the crosscap constraint of [24, 25], that indeed, for a one-dimensional torus, leaves only one 
relative sign between even and odd momentum sums. Notice that all choices of signs in (3.1), that determine the (anti)symmetrization of the states, respect the fusion rules.

This procedure may be generalized to any rational model with $Z_{2}$ automorphisms, where the Klein bottle projections that forbid the introduction of the open sector do not involve massless characters in the transverse channel. In the next section, we shall exploit this possibility to recover the results of [19], but we should mention the simplest instance, toroidal compactification to four dimensions, that gives a string setting to $N=4$ supergravity coupled to six vector multiplets.

\section{Six-Dimensional Models}

In six dimensions there are several possible types of Gepner models [2]. They correspond to toroidal compactifications, to orbifolds of rational tori and to some interacting rational $N=2$ superconformal field theories 2 . The starting point is a "parent" type IIB theory, whose chiral spectrum is uniquely fixed by target-space $N=(2,0)$ supersymmetry. Indeed, aside from (non-chiral) models with extended supersymmetry, that correspond to rational points of toroidal compactifications, any modular invariant torus amplitude results in a massless spectrum including the $N=(2,0)$ supergravity multiplet (the graviton, 5 self-dual tensors and 2 right-handed $(R)$ gravitini) and 21 tensor multiplets (one anti-self-dual tensor, 5 scalars and 2 left-handed $(L)$ tensorini). The scalar fields of the resulting low-energy supergravity parametrize the coset $S O(5,21) / S O(5) \times S O(21)$.

In the open descendants supersymmetry is reduced to $N=(1,0)$, and the unoriented closed spectrum consists of the $N=(1,0)$ supergravity multiplet (the graviton, a self-dual tensor and a $R$ gravitino) coupled to $n_{T}^{c}$ tensor multiplets (an anti-self-dual tensor, a $L$ tensorino and a scalar) and $n_{H}^{c}$ hypermultiplets (four scalars and a $L$ hyperino). The open unoriented sector contains $n_{V}^{o}$ vector multiplets (a vector and a $R$ gaugino) and $n_{H}^{o}$ charged hypermultiplets. It should be appreciated that $n_{T}^{c}+n_{H}^{c}$ is fixed to be 21 since the Klein-bottle projection simply halves the fermi degrees of freedom. The different models

\footnotetext{
${ }^{3}$ More precisely, in this case the $N=2$ superalgebra extends to an $N=4$ superalgebra, that includes an $S U(2)$ subalgebra.
} 
that we describe give rise to different values of both $n_{T}^{c}$ and $n_{H}^{c}$, while the presence of a self-dual tensor in the $N=(1,0)$ supergravity multiplet leaves a net number of $n_{T}^{c}-1$ anti-self-dual tensors. The tensor fields that flow in the transverse channel and correspond to unphysical R-R scalars take part in a generalized GS mechanism [17.

\subsection{The $(k=2)^{4}$ Models with $Z_{2}$ Symmetry}

The first class of models that we discuss is obtained tensoring four copies of the $k=2$ superconformal model. It corresponds to the $Z_{2}$ orbifold of the maximal torus of $S O(8)$ [16]. Each $k=2$ minimal model with $c=3 / 2$ is equivalent to the direct product of the Ising model with $c=1 / 2$ and a free boson $(c=1)$ theory at $R=\sqrt{8}$. Denoting by $\{o, \psi, \sigma\}$ the three characters of the Ising model and by $\rho_{m}, m=-3, \ldots, 4$, the eight characters of the $c=1$ theory one finds that the $N=2$ characters in the NS sector are given by $(o, \psi) \times\left(\rho_{2 p}\right)$ and $\sigma \times\left(\rho_{2 p+1}\right)$ while those in the $\mathrm{R}$ sector are given by $(o, \psi) \times\left(\rho_{2 p+1}\right)$ and $\sigma \times\left(\rho_{2 p}\right)$. The graviton orbit, that gives rise to the identity character, reads

$$
N_{0}^{+}=\left(o \rho_{0}+\psi \rho_{4}\right)^{4}+\left(o \rho_{2}+\psi \rho_{-2}\right)^{4}+\left(o \rho_{-2}+\psi \rho_{2}\right)^{4}+\left(o \rho_{4}+\psi \rho_{0}\right)^{4}
$$

A proper definition of the characters requires the resolution of a fixed-point ambiguity. There are 5 more massless $(h=1 / 2)$ and 10 massive $(h=1)$ characters in one-toone correspondence with those of the first class of models in [13. In this case, the $P=T^{1 / 2} S T^{2} S T^{1 / 2}$ modular transformation that relates direct and transverse Möbius channels [13] is diagonal. In particular, $P_{11}=-1$, and thus only symplectic CP groups are present in the open-string sector. This is in marked contrast with the results of [18, 19]. In order to switch to unitary or orthogonal CP groups, one has to introduce discrete Wilson lines [13], relative phases between crosscap and boundary operators, that break in part the internal symmetry [28]. The CP symmetry is reduced, since a non-vanishing NS-NS antisymmetric tensor is present in the $S O(8)$ torus [14].

An exhaustive analysis of all modular invariant torus amplitudes gives three type IIB parent theories. The spectra of the corresponding open descendants are listed in table 1 for simple choices of CP multiplicities consistent with tadpole cancellation. Non standard 


\begin{tabular}{|c|c|c|c|c|c|c|}
\hline Mod. & $n_{T}^{c}$ & $n_{H}^{c}$ & CP Gauge Group & $n_{V}^{o}$ & Charged Hypermultiplets & $n_{H}^{o}$ \\
\hline $\mathbf{A}_{16}$ & 7 & 14 & $S p(4)^{\otimes 4}$ & 40 & $\begin{array}{c}(\mathbf{4}, \mathbf{1}, \mathbf{4}, \mathbf{1}) \oplus(\mathbf{1}, \mathbf{4}, \mathbf{1}, \mathbf{4}) \\
\oplus 2(\mathbf{4}, \mathbf{1}, \mathbf{1}, \mathbf{4}) \oplus 2(\mathbf{1}, \mathbf{4}, \mathbf{4}, \mathbf{1})\end{array}$ & 96 \\
\hline $\mathbf{B}_{16}$ & 9 & 12 & $S p(4) \otimes S p(4)$ & 20 & $(\mathbf{1 0}, \mathbf{1}) \oplus(\mathbf{1}, \mathbf{1 0})$ & 20 \\
\hline $\mathbf{D}_{16}$ & 5 & 16 & $S p(8)^{\otimes 4}$ & 144 & $\begin{array}{c}(\mathbf{8}, \mathbf{8}, \mathbf{1}, \mathbf{1}) \oplus(\mathbf{1}, \mathbf{1}, \mathbf{8}, \mathbf{8}) \\
\oplus(\mathbf{8}, \mathbf{1}, \mathbf{8}, \mathbf{1}) \oplus(\mathbf{1}, \mathbf{8}, \mathbf{1}, \mathbf{8})\end{array}$ & 256 \\
\hline
\end{tabular}

Table 1: Open descendants of $(k=2)^{4} Z_{2}$ Gepner model.

Klein projections, with only massive characters in the transverse channel, are allowed in all these cases, and give rise to the anomaly free spectrum with $n_{T}^{c}=9$ and $n_{H}^{c}=12$ with no open-string states. This should be contrasted with conventional $B_{16}$ models, where massless tadpoles require that the same closed spectrum be accompanied by anomaly-free open sectors.

\subsection{The $(k=2)^{4}$ Models with $Z_{4}$ Symmetry}

Another class of rational models arises from a $Z_{4}$ orbifold of the $S U(2)^{\otimes 4}$ torust. The type IIB partition function may be written in terms of 64 characters, and corresponds to the second class of models in [13]. In addition to the identity character, there is another massless self-conjugate character, 16 complex massless characters, 2 massive self-conjugate characters with $h=3 / 2$, two sets of 8 massive characters each with $h=3 / 4$ and $h=5 / 4$, respectively, and, finally, 12 self-conjugate and 16 complex massive characters with $h=1$.

An exhaustive analysis of all modular invariant torus amplitudes gives rise to seven $N=(2,0)$ type IIB parent theories. The spectra of the corresponding open descendants are listed in table 2 for simple choices of CP multiplicities consistent with tadpole cancellation. The diagonal $\left(\mathbf{D}_{64}\right)$ and charge-conjugation $\left(\mathbf{C}_{64}\right)$ modular invariants give rise to quite different descendants. The $\mathbf{D}_{64}$ model with standard Klein bottle projection yields a closed-string spectrum with one tensor multiplet and 20 hypermultiplets, while the $\mathbf{C}_{64}$

\footnotetext{
${ }^{4}$ At the closed-string level, this rational model is equivalent to the $Z_{2}$ orbifold of the $S O(8)$ torus [16], but for the absence of a NS-NS antisymmetric tensor background.
} 


\begin{tabular}{|c|c|c|c|c|c|c|}
\hline Mod. & $n_{T}^{c}$ & $n_{H}^{c}$ & CP Gauge Group & $n_{V}^{o}$ & Charged Hypermultiplets & $n_{H}^{o}$ \\
\hline \hline $\mathbf{A}_{64}$ & 5 & 16 & $S p(8) \otimes S p(8)$ & 72 & $(\mathbf{2 8 , 1}) \oplus(\mathbf{1}, \mathbf{2 8}) \oplus 2(\mathbf{8}, \mathbf{8})$ & 184 \\
\hline $\mathbf{B}_{64}$ & 7 & 14 & $S p(8) \otimes S p(8)$ & 72 & $2(\mathbf{8}, \mathbf{8})$ & 128 \\
\hline $\mathbf{C}_{64}$ & 9 & 12 & $U(8) \otimes U(8)$ & 128 & $\left(\mathbf{8}, \mathbf{8}^{*}\right) \oplus\left(\mathbf{8}^{*}, \mathbf{8}\right)$ & 128 \\
\hline $\mathbf{D}_{64}$ & 1 & 20 & $S p(16) \otimes S p(16)$ & 272 & $(\mathbf{1 2 0}, \mathbf{1}) \oplus(\mathbf{1}, \mathbf{1 2 0}) \oplus(\mathbf{1 6}, \mathbf{1 6})$ & 496 \\
\hline $\mathbf{E}_{64}$ & 9 & 12 & $U(8)$ & 64 & $\mathbf{6 4}$ & 64 \\
\hline $\mathbf{F}_{64}$ & 9 & 12 & $U(4) \otimes U(4)$ & 32 & $(\mathbf{1 6}, \mathbf{1}) \oplus(\mathbf{1}, \mathbf{1 6})$ & 32 \\
\hline $\mathbf{G}_{64}$ & 9 & 12 & $U(4)$ & 16 & $\mathbf{1 6}$ & 16 \\
\hline
\end{tabular}

Table 2: Open descendants of $(k=2)^{4} Z_{4}$ Gepner model.

model leads to 9 tensor multiplets and 12 hypermultiplets. Similar results have been found in [18, 19]. In the $\mathbf{C}_{64}$ case, although the closed spectrum is anomaly free, one has to introduce open strings to cancel unphysical tadpoles in the transverse channel. The resulting CP group is at most $S p(16) \otimes S p(16)$ for the $\mathbf{D}_{64}$ case and $U(8) \otimes U(8)$ for the $\mathbf{C}_{64}$ case. The introduction of discrete Wilson lines [13] leads to CP group enhancement to $U(16) \otimes U(16)$ for the $\mathbf{D}_{64}$ model, with the anomaly-free spectrum also found in [18].

\subsection{The $(k=1)^{6}$ Models}

The last class of models that we would like to discuss descends from a $Z_{3}$ orbifold of the maximal torus of $S U(3) \otimes S U(3)$, or equivalently [16] from the tensor product of six copies of the $k=1$ minimal $N=2$ superconformal theory with $c=1$. The latter is equivalent to a free boson at radius $R=\sqrt{12}$. The twelve primary fields have conformal weights $h_{m}=m^{2} / 24$ with $m=-5, \ldots, 6$, and the corresponding characters will be denoted by $\xi_{m}$.

The graviton orbit [16] that gives rise to the identity character

$$
N_{0}^{+}=\left(\xi_{0}+\xi_{6}\right)^{6}+\left(\xi_{2}+\xi_{-4}\right)^{6}+\left(\xi_{4}+\xi_{-2}\right)^{6}
$$

is the only self-conjugate one. Resolving the fixed point ambiguity, one finds 20 more massless characters, and two sets of 30 characters with $h=5 / 6$ and $h=13 / 6$.

For the diagonal model $\left(\mathbf{D}_{81}\right)$, the unoriented closed spectrum resulting from a standard Klein bottle projection includes the supergravity multiplet and one hypermultiplet 
from the identity character. The other 20 massless characters give half hypermultiplet each. The resulting spectrum contains no tensor multiplets, and the dilaton must thus lie in a hypermultiplet. This closed spectrum is anomalous and requires the introduction of open strings. Tadpole cancellation in the transverse channel selects the CP gauge group $S O(8)$ and, aside from the vector multiplet, there are 10 hypermultiplets in the adjoint representation. This representation is anomaly free and the chiral fermion content of the open-string spectrum exactly cancels the gravitational anomaly in the closed-string spectrum. Upon reduction on $T_{2}$ to $D=4$, the theory would not be asymptotically free. It would be very interesting to find a model with no tensor multiplets with an asymptotically free CP group. The analytic prepotential of the resulting effective field theory would not receive spacetime instanton corrections.

The charge conjugation modular invariant $\left(\mathbf{C}_{81}\right)$ leads to an open descendant with 10 hypermultiplets and 10 tensor multiplets arising from massless characters different from the identity, and one hypermultiplet and the supergravity multiplet arising from the identity. The closed spectrum is anomalous and tadpole cancellation requires the introduction of open strings. The gauge group is once again $S O(8)$, but the spectrum contains only the vector multiplet. The open spectrum differs from the one found in 18, 19 for the $Z_{3}$ orbifold, since the Gepner model involves a rank 6 NS-NS antisymmetric tensor background.

An exhaustive analysis of all modular invariant torus amplitudes results in six equivalent type IIB parent theories. The type I spectra are summarized in table 3 for some simple choices of the CP multiplicities. Although it is not evident from the table, $S O(8)$ is always allowed. This is precisely the subgroup of $S O(32)$ preserving a generic configuration of 24 instantons on K3 as required by anomaly cancellation, and suggests an F-theory interpretation for these models. For an elliptic CY 3 -fold $X$ fibered over a base $B$, the number of tensor multiplets is $n_{T}=h_{11}(B)-1$, the rank of the gauge group is $r_{V}=h_{11}(X)-h_{11}(B)-1$ and the number of neutral hypermultiplets is $n_{H}^{0}=h_{12}(X)+1$ [22]. A large class of elliptic CY 3-folds, studied by Voisin and Borcea and classified by Nikulin in terms of three invariants $(r, a, \delta)$, have Hodge numbers $h_{11}(X)=5+3 r-a$ and 


\begin{tabular}{|c|c|c|c|c|c|c|}
\hline Mod. & $n_{T}^{c}$ & $n_{H}^{c}$ & CP Gauge Group & $n_{V}^{o}$ & Charged Hypermultiplets & $n_{H}^{o}$ \\
\hline \hline $\mathbf{A}_{81}$ & 6 & 15 & $U(4)$ & 16 & $4 \times \mathbf{1 6} \oplus 3 \times\left(\mathbf{6} \oplus \mathbf{6}^{*}\right)$ & 100 \\
\hline $\mathbf{B}_{81}$ & 8 & 13 & $U(4)$ & 16 & $2 \times \mathbf{1 6} \oplus \mathbf{6} \oplus \mathbf{6}^{*}$ & 44 \\
\hline $\mathbf{C}_{81}$ & 10 & 11 & $S O(8)$ & 28 & - & 0 \\
\hline $\mathbf{D}_{81}$ & 0 & 21 & $S O(8)$ & 28 & $10 \times \mathbf{2 8}$ & 280 \\
\hline $\mathbf{E}_{81}$ & 10 & 11 & $S O(8)$ & 28 & - & 0 \\
\hline $\mathbf{F}_{81}$ & 10 & 11 & $S O(8)$ & 28 & - & 0 \\
\hline
\end{tabular}

Table 3: Open descendants of $(k=1)^{6}$ Gepner model.

$h_{12}(X)=65-3 r-2 a$. F-theory compactification on these spaces leads to an $S O(8)^{k+1}$ gauge group, with $k=(r-a) / 2$, and to $g=(22-r-a) / 2$ adjoint hypermultiples. It is tempting to conjecture that the type I models with $G=S O(8)$ correspond to the choice $r=a=n_{T}^{c}+1$.

\section{Anomaly Cancellation and Final Remarks}

Aside from $U(1)$ anomalies, tadpole cancellations guarantee the absence of gauge and gravitational anomalies for all the models discussed so far. Indeed, the antisymmetric tensor fields that flow in the transverse channel take part in a generalized GS mechanism [17] that allows the cancellation of not necessarily factorized 6D chiral anomalies. In table 4 we report the anomaly polynomials for the models discussed in the previous section. These expressions determine the kinetic terms of the gauge fields and their singularities at finite coupling [17], that have been associated to phase transitions [27] with tensionless strings [11]. Antisymmetric tensor fields, however, can not cancel chiral anomalies for abelian gauge groups with charged hypermultiplets. It has been proposed [29, [1] that a mechanism similar to the one taking place in $\mathrm{D}=4$ be at work in this case. In six dimensions the relevant field is a 4 -form potential, dual to a pseudo-scalar. The massless type I spectra that we have discussed include several R-R scalars. These admit exact Peccei-Quinn symmetries and may be dualized into 4-form potentials, that can couple to abelian fields via $A_{4} \wedge d A_{1}$. In the presence of chiral anomalies, these R-R fields could 


\begin{tabular}{|c|c|}
\hline Model & Anomaly Polynomial \\
\hline $\mathbf{A}_{16}$ & $\begin{array}{l}-\frac{1}{16}\left(\frac{1}{2} \operatorname{tr} R^{2}-\operatorname{tr} F_{1}^{2}-\operatorname{tr} F_{2}^{2}-\operatorname{tr} F_{3}^{2}-\operatorname{tr} F_{4}^{2}\right)^{2} \\
+\frac{1}{8}\left(\operatorname{tr} F_{1}^{2}+\operatorname{tr} F_{2}^{2}-\operatorname{tr} F_{3}^{2}-\operatorname{tr} F_{4}^{2}\right)^{2}+\frac{1}{16}\left(\operatorname{tr} F_{1}^{2}-\operatorname{tr} F_{2}^{2}+\operatorname{tr} F_{3}^{2}-\operatorname{tr} F_{4}^{2}\right)^{2}\end{array}$ \\
\hline $\mathbf{D}_{16}$ & $\begin{array}{l}-\frac{1}{32}\left(\operatorname{tr} F_{1}^{2}+\operatorname{tr} F_{2}^{2}+\operatorname{tr} F_{3}^{2}+\operatorname{tr} F_{4}^{2}+\operatorname{tr} R^{2}\right)^{2}+\frac{3}{32}\left(\operatorname{tr} F_{1}^{2}-\operatorname{tr} F_{2}^{2}-\operatorname{tr} F_{3}^{2}+\operatorname{tr} F_{4}^{2}\right)^{2} \\
+\frac{1}{32}\left(\operatorname{tr} F_{1}^{2}+\operatorname{tr} F_{2}^{2}-\operatorname{tr} F_{3}^{2}-\operatorname{tr} F_{4}^{2}\right)^{2}+\frac{1}{32}\left(\operatorname{tr} F_{1}^{2}-\operatorname{tr} F_{2}^{2}+\operatorname{tr} F_{3}^{2}-\operatorname{tr} F_{4}^{2}\right)^{2}\end{array}$ \\
\hline $\mathbf{A}_{64}$ & $-\frac{1}{8}\left(\frac{1}{2} \operatorname{tr} R^{2}-\operatorname{tr} F_{1}^{2}-\operatorname{tr} F_{2}^{2}\right)^{2}+\frac{1}{8}\left(\operatorname{tr} F_{1}^{2}-\operatorname{tr} F_{2}^{2}\right)^{2}$ \\
\hline $\mathbf{B}_{64}$ & $-\frac{1}{16}\left(\frac{1}{2} \operatorname{tr} R^{2}-\operatorname{tr} F_{1}^{2}-\operatorname{tr} F_{2}^{2}\right)^{2}+\frac{3}{16}\left(\operatorname{tr} F_{1}^{2}-\operatorname{tr} F_{2}^{2}\right)^{2}$ \\
\hline $\mathrm{C}_{64}$ & $\frac{1}{4}\left(\operatorname{tr} F_{1}^{2}-\operatorname{tr} F_{2}^{2}\right)^{2}+\frac{1}{3}\left(\operatorname{tr} F_{1}-\operatorname{tr} F_{2}\right)\left[\left(\operatorname{tr} F_{1}^{3}-\operatorname{tr} F_{2}^{3}\right)-\frac{1}{16} \operatorname{tr} R^{2}\left(\operatorname{tr} F_{1}-\operatorname{tr} F_{2}\right)\right]$ \\
\hline $\mathbf{D}_{64}$ & $-\frac{1}{16}\left(\operatorname{tr} R^{2}-\operatorname{tr} F_{1}^{2}-\operatorname{tr} F_{2}^{2}\right)^{2}+\frac{1}{16}\left(\operatorname{tr} F_{1}^{2}-\operatorname{tr} F_{2}^{2}\right)^{2}$ \\
\hline $\mathbf{A}_{81}$ & $-\frac{3}{8}\left(\frac{1}{4} \operatorname{tr} R^{2}+2 \operatorname{tr} F^{2}\right)^{2}+\frac{1}{8}\left(\operatorname{tr} R^{2} \operatorname{tr} F-16 \operatorname{tr} F^{3}\right) \operatorname{tr} F$ \\
\hline $\mathbf{B}_{81}$ & $-\frac{1}{8}\left(\frac{1}{4} \operatorname{tr} R^{2}-2 \operatorname{tr} F^{2}\right)^{2}+\frac{1}{24}\left(\operatorname{tr} R^{2} \operatorname{tr} F-16 \operatorname{tr} F^{3}\right) \operatorname{tr} F$ \\
\hline $\mathrm{C}_{81}$ & $\frac{1}{8}\left(\frac{1}{4} \operatorname{tr} R^{2}-\operatorname{tr} F^{2}\right)^{2}$ \\
\hline $\mathbf{D}_{81}$ & $-\frac{9}{8}\left(\frac{1}{4} \operatorname{tr} R^{2}-\operatorname{tr} F^{2}\right)^{2}$ \\
\hline $\mathbf{E}_{81}$ & $\frac{1}{8}\left(\frac{1}{4} \operatorname{tr} R^{2}-\operatorname{tr} F^{2}\right)^{2}$ \\
\hline $\mathbf{F}_{81}$ & $\frac{1}{8}\left(\frac{1}{4} \operatorname{tr} R^{2}-\operatorname{tr} F^{2}\right)^{2}$ \\
\hline
\end{tabular}

Table 4: Non-vanishing anomaly polynomials for the models in tables $1,2,3$, with $F_{i}$ the field strength of the $i$-th factor in the CP group. $R$ is the curvature 2-form, and tr denotes the trace in the fundamental representation.

then provide the longitudinal degrees of freedom needed to exclude the (massive) abelian vector fields from the low-energy spectrum, much in the same way as in $D=4$ [30, [10].

The list of 6D Gepner models is clearly not exhausted by these simple cases, and a more complete analysis will be reported in [28]. We have also constructed the simplest 4D Gepner model with $N=1$ supersymmetry, $(k=1)^{9}$. Since it is related to the $Z_{3}$ orbifold of the maximal torus of $S U(3)^{\otimes 3}$, the CP multiplicities are reduced [14 by the non-vanishing (quantized) NS-NS antisymmetric tensor background. The spectrum is encoded in 2187 characters. Apart from the identity, only 168 of them are massless. There are many torus amplitudes that give rise to different descendants. In particular, the type IIB theory based on the charge conjugation invariant may be related to the compactification on a CY threefold with Hodge numbers $h_{11}=84$ and $h_{21}=0$. The open descendant has $84+1$ chiral multiplets in the unoriented closed spectrum and an $S p(4)$ $\mathrm{CP}$ group with 84 chiral multiplets in the adjoint representation in the open spectrum. 
In the diagonal case one finds the same closed spectrum and CP group without charged matter, while the type IIB spectrum, with $84 N=2$ vector multiplets and one universal hypermultiplet, may be related to the mirror of the above threefold.

\section{References}

[1] A. Sen, Nucl. Phys. B278 (1986) 289;

T. Banks, L.J. Dixon, D. Friedan and E. Martinec, Nucl. Phys. B299 (1988) 613.

[2] D. Gepner, Nucl. Phys. B296 (1988) 757, in Proc. 1988 Trieste Spring School on Superstrings.

[3] E. Martinec, Phys. Lett. B217 (1989) 431; B.R. Greene, C. Vafa and N.P. Warner, Nucl. Phys. B324 (1989) 371; C. Vafa and N.P. Warner, Phys. Lett. B218 (1989) 51; W. Lerche, C. Vafa and N.P. Warner, Nucl. Phys. B324 (1989) 427.

[4] See: Essays on Mirror Manifolds, ed. S.-T. Yau (International Press, 1992).

[5] S. Ferrara, J. Harvey, A. Strominger and C. Vafa, Phys. Lett. B361 (1995) 59.

[6] S. Kachru and C. Vafa, Nucl. Phys. B450 (1995) 69; S. Kachru, A. Klemm, W. Lerche, P. Mayr and C. Vafa, Nucl. Phys. B459 (1996) 537.

[7] E. Witten, Nucl. Phys. B443 (1995) 85;

J. Polchinski and E. Witten, Nucl. Phys. B460 (1996) 525.

[8] A. Tseytlin, Phys. Lett. B367 (1996) 84; Nucl. Phys. B467 (1996) 383.

[9] C. Vafa and E. Witten, hep-th/9507050; E. Witten, Nucl. Phys. 460 (1996) 541.

[10] C. Angelantonj, M. Bianchi, G. Pradisi, A. Sagnotti and Ya.S. Stanev, hepth/9606169.

[11] N. Seiberg and E. Witten, hep-th/9603003; M. Berkooz, R.G. Leigh, J. Polchinski, J.H. Schwarz, N. Seiberg and E. Witten, hep-th/9605184.

[12] A. Sagnotti, in "Non-Perturbative Quantum Field Theory", eds. G. Mack et al (Pergamon Press, 1988), p. 521;

P. Hořava, Nucl. Phys. B327 (1989) 461, Phys. Lett. B231 (1989) 251. 
[13] M. Bianchi and A. Sagnotti, Phys. Lett. B247 (1990) 517; Nucl. Phys. B361 (1991) 519.

[14] M. Bianchi, G. Pradisi and A. Sagnotti, Nucl. Phys. B376 (1991) 365.

[15] L. Dixon, J. Harvey, C. Vafa and E. Witten, Nucl. Phys. B261 (1985) 678, B274 (1986) 285.

[16] T. Eguchi, H. Ooguri, A. Taormina and S-K. Yang, Nucl. Phys. B315 (1989) 193.

[17] A. Sagnotti, Phys. Lett. B294 (1992) 196.

[18] E. Gimon and J. Polchinski, hep-th/9601038; A. Dabholkar and J. Park, hepth/9602030.

[19] E. Gimon and C.V. Johnson, hep-th/9604129; A. Dabholkar and J. Park, hepth/9604178.

[20] G. Pradisi and A. Sagnotti, Phys. Lett. B216 (1989) 59.

[21] C. Vafa, hep-th/9602022.

[22] D.R. Morrison and C. Vafa, hep-th/9602114, hep-th/9603161.

[23] A. Sen, hep-th/9605150; K. Dasgupta and S. Mukhi, hep-th/9606044.

[24] D. Fioravanti, G. Pradisi and A. Sagnotti, Phys. Lett. B321 (1994) 349.

[25] G. Pradisi, A. Sagnotti and Ya.S. Stanev, Phys. Lett. B354 (1995) 279, B356 (1995) 230, B381 (1996) 97.

[26] M. Bianchi, S. Ferrara, G. Pradisi, A. Sagnotti and Ya.S. Stanev, hep-th/9607105.

[27] M.J. Duff, R. Minasian and E. Witten, hep-th/9601036.

[28] C. Angelantonj, M. Bianchi, G. Pradisi, A. Sagnotti, Ya.S. Stanev, in preparation.

[29] S. Ferrara, R. Minasian and A. Sagnotti, hep-th/9604097.

[30] E. Witten, Phys. Lett. B149 (1984) 351;

M. Dine, N. Seiberg and E. Witten, Nucl. Phys. B289 (1987) 317. 